\title{
Comparison of 2D Fat Suppressed Proton Density (FS-PD) and 3D (WATS-c) MRI pulse sequences in evaluation of chondromalacia patellae
}

\author{
Ahmed Ibrahim Tawfik ${ }^{*}$, Wael Hamza Kamr and Saher Ebrahim Taman
}

\begin{abstract}
Background: Comparing the diagnostic performance of widely used 2D FSE technique (fat-suppressed proton density; FS-PD) and the 3D technique (water-selective cartilage scan; WATS-c) in evaluation of the chondromalacia patella by using arthroscopy as reference standard

Results: Seventy-five adult patients were enrolled in this study. They underwent MRI examinations then arthroscopy done in 2-4 days after it. MRI was done using 2D (FS-PD) and 3D (WATS-c) sequences and MR images were compared by two radiologists separately, then grading of the cartilage lesions was performed according to modified Noyes grading system and comparison between grade $0-1,2$, and 3 lesions was done using arthroscopic findings as a reference. A false-negative result is considered if there was undergrading of chondromalacia and falsepositive result if chondromalacia was overgraded. Each sequence sensitivity, specificity, and accuracy was calculated by both readers.

For reader 1, the sensitivity is 69\% for WATS-c and 80\% for FS-PD and the accuracy is $90 \%$ for WATS-c and $92 \%$ for FS-PD and for reader 2, the sensitivity is $56 \%$ for WATS-c and $84 \%$ for FS-PD and the accuracy is $88 \%$ for WATS-C and $94 \%$ for FS-PD.
\end{abstract}

Conclusion: 2D FS-PD images showed better diagnostic performance than 3D WATS-c images for evaluating chondromalacia patella.

Keywords: WATS-c (water-selective cartilage scan), FS-PD (fat-suppressed proton density), Chondromalacia patella

\section{Background}

Chondromalacia patellae is a common patellar cartilage disorder which cannot be diagnosed only on the basis of taking the patient's symptoms or doing physical examination. Although arthroscopy is the corner stone for diagnosis of chondromalacia patella, magnetic resonance imaging (MRI) is very helpful as a non-invasive method in the diagnosis of patellar chondral lesions $[1,2]$. Increasing magnet strength and using a specialized coil increase both sensitivity and specificity in the diagnosis of chondromalacia. In MRI imaging, we have many pulse sequences used for the evaluation of patellar cartilage, one of the ideal pulse sequences is fat-suppressed proton

\footnotetext{
*Correspondence: ahmedtawfik82@yahoo.com

Diagnostic Radiology Department, Mansoura University, Mansoura, Egypt
}

density (FS-PD) which is very valuable in evaluating patellar cartilage as it has high spatial resolution, suitable contrast-to-noise (CNR) ratio, and good scan time $[3,4]$. Recently, 3D imaging sequences appear to have a role for diagnosis. 3D T1-fast field echo is one of them that use cartilage water excitation (water-selective cartilage scan; WATS-c). It induces fat suppression by using water excitation [5, 6]. FS-PD sequence has an excellent performance in detecting lesions of patellar cartilage [7, 8], but the $3 \mathrm{D}$ sequences are superior in having much thinner slices than that of the 2D images that also gives good diagnostic value, so comparison between the two sequences is valuable in evaluating efficiency of each one in patellar cartilage diagnosis in cases of chondromalacia patella $[9,10]$. 
This prospective study is based on the comparison between the diagnostic performance of widely used 2D fast spin echo (FSE) technique (FS-PD) and the 3D FFE technique (WATS-c) in evaluation of the chondromalacia patella by using arthroscopy as reference standard.

\section{Methods \\ Patients}

Between May 2016 and June 2017, 100 patients underwent MR knee examination were included in the study.

Patient with non-specific knee pain, clinically diagnosed chondromalacia patella, meniscopathy, history of trauma, and osteoarthritic changes are included; twentyfive patients who had tumors, had previous implants, or underwent surgery and patients with motion artifact in MRI images were excluded. Finally, MRI examinations of 75 adult patients ( 36 women patients and 39 men patients, their mean age 35 years; range, $16-76$ years) were enrolled in this study. They underwent MRI examinations then arthroscopy done in 2-4 days after it. Approval was obtained for this prospective study by the institutional review board and all patients gave informed consent before doing MRI acquisition.

\section{MRI examination}

MRI examinations of the patellar cartilage was done using knee coil on 1.5-T MRI scanner (Intera; Philips Medical Systems; Best, The Netherlands) by using a 16 channel transmit/receive special dedicated knee coil, the patients were in the supine position. The 2D FSE fatsuppressed proton density (FS-PD) were obtained in axial images, the 3D T1 FFE with water excitation (WATS-c) sequence were also obtained in axial images. Table 1 shows the imaging protocols.

Table $1 \mathrm{MRI}$ pulse sequences used in this study

\begin{tabular}{lll}
\hline & FS-PD & WATs-C \\
\hline TR & 3000 & 20 \\
TE & 30 & 8 \\
FA & 90 & 25 \\
Scan mode & $2 \mathrm{D}$ & $3 \mathrm{D}$ \\
Technique & FSE & FFE \\
Slice thickness & $1.5 \mathrm{~mm}$ & $1.5 \mathrm{~mm}$ \\
Interslice gap & $0.15 \mathrm{~mm}$ & 0 \\
FOV & $160 \times 160 \times 80$ & $130 \times 130 \times 75$ \\
Matrix & $288 \times 244$ & $256 \times 195$ \\
Time & $2: 54$ & $4: 21$ \\
\hline
\end{tabular}

FA flip angle, FFE fast field echo image, FOV field of view, FS-PD fat-suppressed proton density-weighted image, TE echo time, TR repetition time, WATS-C water-selective cartilage scan

\section{Evaluation of MR images}

After doing MRI pulse sequences with data acquisition of all patients' examinations, obtained MR images were studied and compared by the two radiologists separately and independently.

The grading of the cartilage lesions was performed according to their MRI appearance and following the modified Noyes grading system [11, 12] (Table 2): grade 0 , denoting normal appearance of cartilage (homogeneous signal intensity of the cartilage, intact, its surface without fissuring, and normal cartilaginous thickness); grade 1, there is increased T2 signal intensity of morphologically normal cartilage not oriented at $55^{\circ}$ to the external magnetic field; grade $2 \mathrm{~A}$, there is superficial cartilaginous partial-thickness defect which is less than $50 \%$ of the total cartilaginous articular surface thickness; grade $2 \mathrm{~B}$, there is cartilaginous deep partial-thickness defect which is more than $50 \%$ of the total cartilaginous articular surface thickness; and grade 3 , there is fullthickness defect of cartilage.

Evaluation of patellar surface cartilage was done in four areas, i.e., upper part and lower part in both medial and lateral patellar articular facets. Recording of highest grade of each segment was done, and then arthroscopy was performed about 2 to 4 days after doing MRI.

By using the modified Noyes grading system, comparison between MRI grade $0-1,2$, and 3 lesions was done using arthroscopic findings as reference. A false-negative result is considered if there was undergrading of chondromalacia on the MRI examination, false-positive result if chondromalacia was overgraded.

\section{Statistical analysis}

Sensitivity, specificity, and accuracy of each MRI sequence for detection of patellar cartilage lesion were calculated by both readers.

\section{Results}

A total of 75 patients (M:F $=36: 39$; mean age, 35 years; range, $16-76$ years) were included in our study.

Twenty patients were grade 0,28 were grade 1 lesions, 17 were grade 2 lesions, and 10 patients were grade 3 lesions were diagnosed by arthroscope. In MRI study, all grade 3 lesions were easily depicted by the two readers in each single pulse sequence, so there was statistical difference only in detection of grade 1 and 2 lesions (Figs. 1 and 2) and no significant difference in diagnosis of grade 3 (Fig. 3).

For reader 1 , the sensitivity is $69 \%$ for WATS-c and $80 \%$ for FS-PD, the specificity is $100 \%$ for WATS-c and $96 \%$ for FS-PD, and the accuracy is $90 \%$ for WATS-c and $92 \%$ for FS-PD.

For reader 2, the sensitivity is 56\% for WATS-c and $84 \%$ for FS-PD, the specificity is $100 \%$ for WATS-c and 
Table 2 Modified Noyes grading system for cartilage grading according to MRI abnormalities appearance [11, 12]

\begin{tabular}{ll}
\hline Grade & Description \\
\hline Grade 0 & Normal cartilage \\
Grade 1 & There is increased T2 signal intensity of morphologically normal cartilage not oriented at $55^{\circ}$ to the external magnetic field \\
Grade 2A & There is superficial partial-thickness cartilage defect less than $50 \%$ of total articular surface thickness \\
Grade 2B & There is deep partial-thickness cartilage defect more than $50 \%$ of total articular surface thickness \\
Grade 3 & Full-thickness cartilage defect \\
\hline
\end{tabular}

$98 \%$ for FS-PD, and the accuracy is $88 \%$ for WATS-c and $94 \%$ for FS-PD (Fig. 4).

\section{Discussion}

Proton density (PD) sequence has been used in the evaluation of knee cartilage with good signal to noise ratio (SNR) comparable to other sequences. Furthermore, fat suppression increases cartilage lesions depiction, The FSPD pulse sequence is very sensitive for depiction of chondromalacia patella and not only increases the accuracy in detection of cartilage lesions but also increases it in detection of underlying bony lesions in grade 3 chondromalacia patella $[13,14]$. As fat-saturated proton density has high spatial resolution in a suitable scan time, it has been considered very efficient in evaluating patellar cartilage. Yoshioka et al. [15] reported that there is a higher signal to noise (SNR) in FS-PD than short tau inversion recovery (STIR) in articular cartilage imaging [16].

The 3D GRE pulse sequences have good images with high spatial resolution due to isotropic voxels; they provide better evaluation of articular cartilage but they are not optimal in assessment of subchondral bone. This assessment is very important in detecting the extent of both degenerative and traumatic cartilaginous lesions [17].

New sequences, which helped us to obtain good images of the patellar cartilage with both high isotropic spatial resolution and contrast resolution, like 3D intermediate-weighted FSE sequences which depend on changing flip angle and reduce time of acquisition that provide isotropic spatial resolution allow multiplanar reformatting with high quality that allows for good depiction of any lesions in subchondral bone [18].

Recently, many 3D sequences appear to be valuable and play a role in cartilage imaging and detecting cartilage lesions. A comparison was done in-between these new sequences and conventional 2D images. More recently, fat-suppression isotropic 3D sequences such as WATS-c appear and have to be included in the search to reach optimal image sequences for diagnosis [19, 20].

As it is less sensitive to magnetic field inhomogeneity, WATS-c sequence has the capacity to suppress fat signal much better than other presaturation techniques [21].

Most of studies done for evaluation of 3D MRI sequences have either surgical reference or arthroscopic reference standards, their aim was to qualitatively compare between these new sequences and the older $2 \mathrm{D}$ sequences FS-PD using multiple observers [22, 23].

It was reported by Schaefer et al. [8] that there were underestimation of grade 1 cartilage lesions on the $3 \mathrm{D}$ pulse sequences and there were poor internal contrast between intact cartilage and chondromalacia.

In our study, arthroscopic findings were used as a reference in comparing sensitivity, specificity, and accuracy of 2D FS-PD and 3D WATS-c by two radiologists.

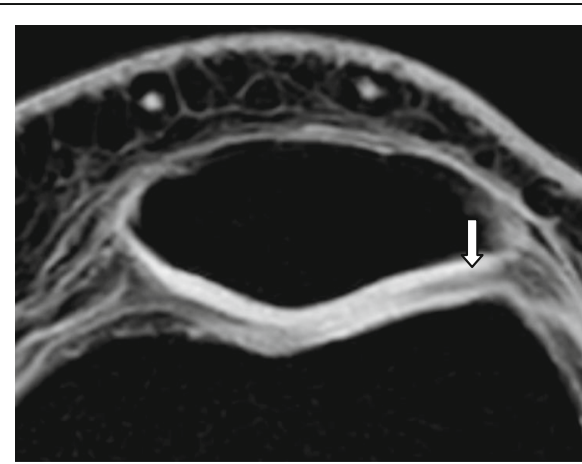

(A)

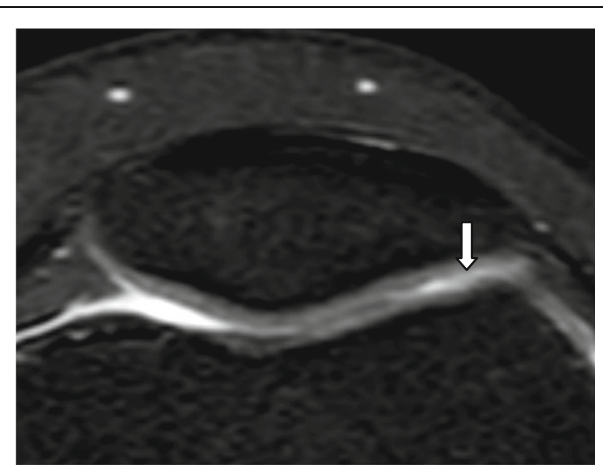

(B)

Fig. 1 Thirty-three-year-old man with knee pain, 3D WATC-s image (a) showing normal cartilage thickness displaying bright signal intensity on WATS-c image with superficial linear area of low signal intensity in lateral patellar articular facet in keeping with grade 1 chondromalacia. FS-PD image (b) show corresponding linear area of abnormal high signal intensity on low signal of cartilage with reversing signal seen in WATS-c sequence 


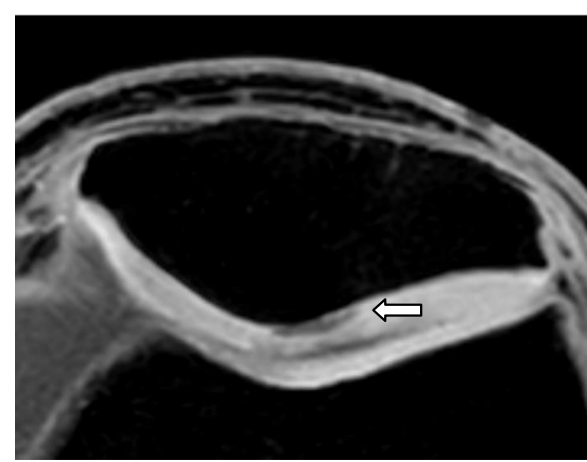

(A)

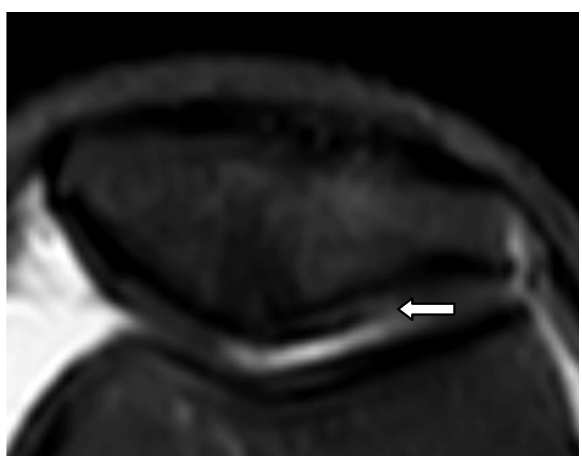

(B)

Fig. 2 Forty-five-year-old woman with knee pain, 3D WATS-c (a) showing linear areas of low signal intensity in lateral patellar articular facet and its surface less than $50 \%$ of articular thickness in keeping with grade 2A, FS-PD (b) show corresponding high signal intensity

In our study, sensitivity of FS-PD is higher than that of WATS-c as it is $80 \%$ and $69 \%$, respectively, by one reader and $84 \%$ and $56 \%$, respectively, by the other one; also, accuracy of FS-PD is higher than that of WATS-c as it is $92 \%$ and $90 \%$, respectively, by one reader and the other one $94 \%$ and $88 \%$, respectively; these results are coping with recent studies showed that FS-PD had a higher sensitivity and accuracy for cartilage pathology depiction than WATS-c did $[9,16,22]$ as the abnormality found in internal cartilage is hidden by the presence of high signal intensity seen in WATS-c images and there is also low contrast between synovial fluid and cartilage that made it difficult to detect early pathological changes like cartilage fissures or fibrillation [17, 24, 25].

There is difficulty in evaluation of patellar cartilage lesions by both routine and cartilage-specific MRI sequences [26], In our study, WATS-c had both higher signal to noise and contrast to noise ratios than that of

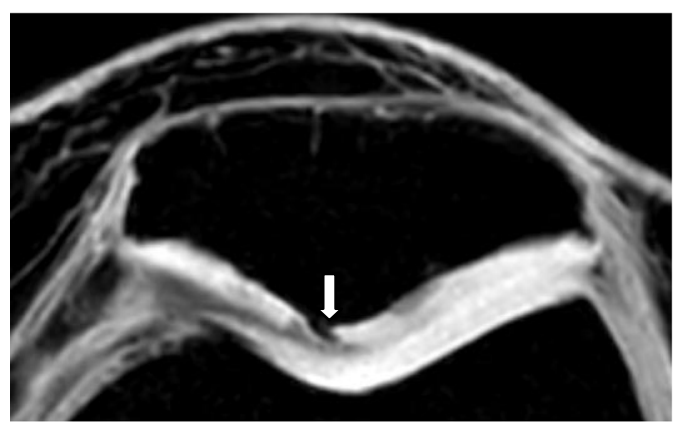

(A)

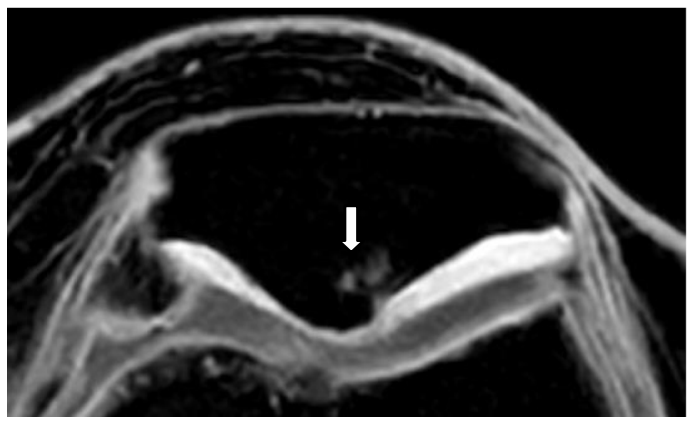

(B)

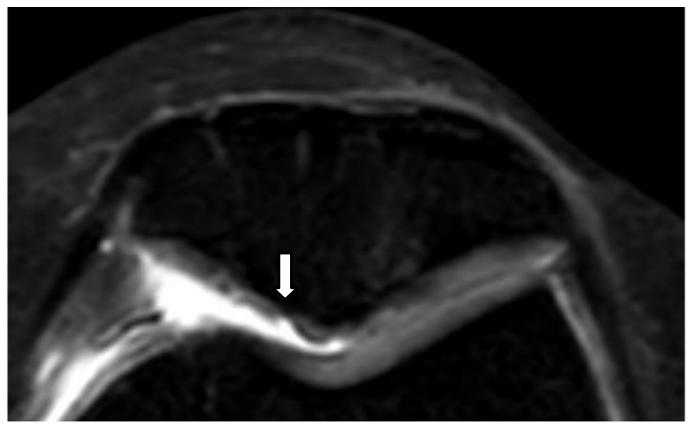

(C)

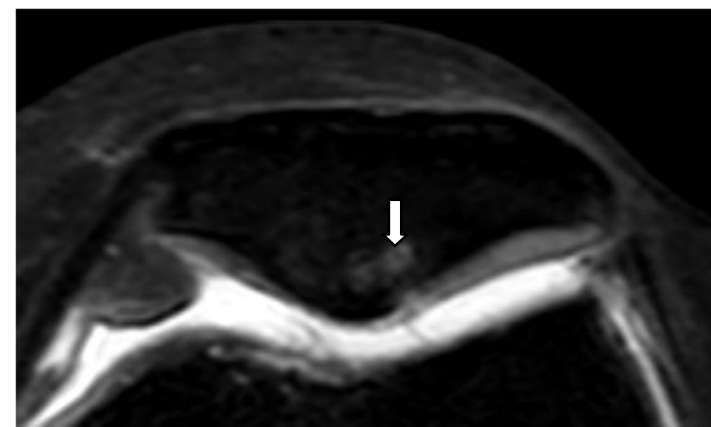

(D)

Fig. 3 Sixty-eight-year-old woman with knee pain, two consecutive axial images of 3D WATS-c (a, b) showing multiple areas of low signal intensity in medial and lateral patellar articular facet with areas of full-thickness cartilage defect and underlying bony erosion in keeping with grade 3. Corresponding two consecutive images of FS-PD (c, d) show high signal intensity in cartilage and associated underlying bony erosion 


\section{- Sensitivity $\quad$ Specificity $\quad$ Accuracy}

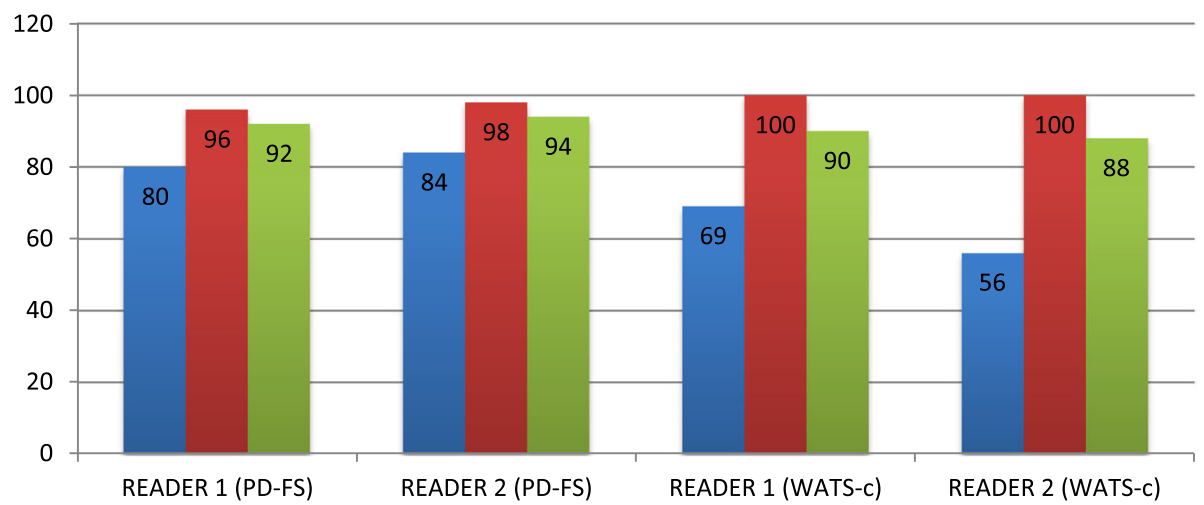

Fig. 4 Sensitivity, specificity, and accuracy of sequences by two readers

FS-PD [27] However, there is limitation of soft-tissue contrast as it has low T1/T2 values [28], and in our study, it is limited in seeing the cartilage substance for chondromalacia detection and also there is difficulty in surface irregularity detection due to joint fluid.

\section{Conclusion}

FS-PD images showed better diagnostic performance than 3D WATS-c images for evaluating chondromalacia patella.

\section{Abbreviations}

3D MRI: Three-dimensional magnetic resonance imaging; FSE: Fast spin echo; FS-PD: Fat-suppressed proton density; WATS-c: Water-selective cartilage scan

\section{Acknowledgements}

Not applicable

\section{Authors' contributions}

AT contributed to the study concepts, study design, data acquisition, and manuscript preparation. WK contributed to the quality control of data and algorithms and manuscript review. AT and WK contributed to statistical analysis. ST contributed to the data analysis and interpretation and manuscript editing. All authors read and approved the final manuscript.

\section{Funding}

Not applicable

\section{Availability of data and materials}

The datasets used and analyzed during the current study are available from the corresponding author on reasonable request.

\section{Ethics approval and consent to participate}

This study was approved by Research Ethics Committee of Almana General Hospital, Saudi Arabia, on 21 April 2016 (reference number not available) All patients included in this study (all above 16 years old) gave written informed consent to participate in this research.

\section{Consent for publication}

All patients included in this research (all above 16 years old) gave written informed consent to publish the data contained within this study.

\section{Competing interests}

The authors declare that they have no competing interests.
Received: 26 November 2019 Accepted: 2 December 2019

Published online: 16 December 2019

\section{References}

1. Outerbridge RE (1961) The etiology of chondromalacia patellae. J Bone Joint Surg Br 43(4):752-757

2. Pihlajamäki HK, Kuikka PI, Leppänen W, Kiuru MJ, Mattila VM (2010) Reliability of clinical findings and magnetic resonance imaging for the diagnosis of chondromalacia patellae. J Bone Joint Surg Am 92(4):927-934

3. Gold GE, Chen CA, Koo S, Hargreaves BA, Bangerter NK (2009) Recent advances in MRI of articular cartilage. Am J Roentgenol 193(3):628-638

4. Crema MD, Roemer FW, Marra MD, Burstein D, Gold GE, Eckstein F et al (2011) Articular cartilage in the knee: current MR imaging techniques and applications in clinical practice and research. Radiographics 31(1):37-62

5. Mohr A, Priebe M, Taouli B, Grimm J, Heller M, Brossmann J (2003) Selective water excitation for faster MR imaging of articular cartilage defects: initial clinical results. Eur Radiol 13:686-689

6. Hauger O, Dumont E, Chateil JF, Moinard M, Diard F (2002) Water excitation as an alternative to fat saturation in MR imaging: preliminary results in musculoskeletal imaging. Radiology 224:657-663

7. Saadat E, Jobke B, Chu B, Lu Y, Cheng J, Li X et al (2008) Diagnostic performance of in vivo 3-T MRI for articular cartilage abnormalities in human osteoarthritic knees using histology as standard of reference. Eur Radiol 18:2292-2302

8. Schaefer FK, Kurz B, Schaefer PJ, Fuerst M, Hedderich J, Graessner J et al (2007) Accuracy and precision in the detection of articular cartilage lesions using magnetic resonance imaging at 1.5 Tesla in an in vitro study with orthopedic and histopathologic correlation. Acta Radiol 48:1131-1137

9. Bauer JS, Barr C, Henning TD, Malfair D, Ma CB, Steinbach L et al (2008) Magnetic resonance imaging of the ankle at 3.0 Tesla and 1.5 Tesla in human cadaver specimens with artificially created lesions of cartilage and ligaments. Investig Radiol 43:604-611

10. Hardy PA, Recht MP, Piraino DW (1998) Fat suppressed MRI of articular cartilage with a spatial-spectral excitation pulse. J Magn Reson Imaging 8: 1279-1287

11. Kijowski R, Blankenbaker DG, Davis KW, Shinki K, Kaplan LD, Smet AAD (2009) Comparison of 1.5- and 3.0-T MR imaging for evaluating the articular cartilage of the knee joint1. Radiology 250(3):839-848

12. Gagliardi JA, Chung EM, Chandnani VP, Kesling KL, Christensen KP, Null RN et al (1994) Detection and staging of chondromalacia patellae: relative efficacies of conventional MR imaging, MR arthrography, and CT arthrography. AJR Am J Roentgenol 163(3):629-636

13. Sonin AH, Pensy RA, Mulligan ME, Hatem S (2002) Grading articular cartilage of the knee using fast spin-echo proton densityweighted MR imaging without fat suppression. AJR Am J Roentgenol 179:1159-1166

14. Mohr A, Roemer FW, Genant HK, Liess C (2003) Using fat-saturated proton density-weighted MR imaging to evaluate articular cartilage. AJR Am J Roentgenol 181:280-281 
15. Yoshioka H, Stevens K, Hargreaves BA, Steines D, Genovese M, Dillingham MF et al (2004) Magnetic resonance imaging of articular cartilage of the knee: comparison between fat-suppressed threedimensional SPGR imaging, fat-suppressed FSE imaging, and fatsuppressed three-dimensional DEFT imaging, and correlation with arthroscopy. J Magn Reson Imaging 20:857-864

16. Ristow O, Steinbach L, Sabo G, Krug R, Huber M, Rauscher I et al (2009) Isotropic 3D fast spin-echo imaging versus standard $2 \mathrm{D}$ imaging at $3.0 \mathrm{~T}$ of the knee - image quality and diagnostic performance. Eur Radiol 19(5): 1263-1272

17. Friedrich KM, Reiter G, Kaiser B, Mayerhöfer M, Deimling M, Jellus V et al (2011) High-resolution cartilage imaging of the knee at 3T: basic evaluation of modern isotropic 3D MR-sequences. Eur J Radiol 78(3):398-405

18. Zur Y (2000) Design of improved spectral-spatial pulses for routine clinical use. Magn Reson Med 43:410-420

19. Recht MP, Piraino DW, Paletta GA, Schils JP, Belhobek GH (1996) Accuracy of fat-suppressed three-dimensional spoiled gradient-echo FLASH MR imaging in the detection of patellofemoral articular cartilage abnormalities. Radiology 198(1):209-212

20. Duc SR, Koch P, Schmid MR, Horger W, Hodler J, Pfirrmann CWA (2007) Diagnosis of articular cartilage lesions of the knee: prospective clinical evaluation of a 3D water-excitation true FISP sequence. Radiology 243(2): 475-482

21. Mohr A (2003) The value of water-excitation 3D FLASH and fatsaturated PDW TSE MR imaging for detecting and grading articular cartilage lesions of the knee. Skelet Radiol 32:396-402

22. Yoshioka H, Stevens K, Genovese M, Dillingham MF, Lang P (2004) Articular cartilage of knee: normal patterns at MR imaging that mimic disease in healthy subjects and patients with osteoarthritis. Radiology 231:31-38

23. Hargreaves BA, Gold GE, Beaulieu CF, Vasanawala SS, Nishimura DG, Pauly JM (2003) Comparison of new sequences for high-resolution cartilage imaging. Magn Reson Med 49:700-709

24. Scheffler K (2004) Fast frequency mapping with balanced SSFP: theory and application to proton-resonance frequency shift thermometry. Magn Reson Med 51:1205-1211

25. Vlaardingerbroek MT, den Boer JA (2003) Magnetic resonance imaging: theory and practice, 3rd edn. Springer, New York

26. Van Dyck P, Kenis C, Vanhoenacker FM, Lambrercht V, Wouters K, Gielen JL et al (2014) Comparison of 1.5- and 3-T MR imaging for evaluating the articular cartilage of the knee. Knee Surg Sports Traumatol Arthrosc 22: 1376-1384

27. Roemer FW, Hunter DJ, Guermazi A (2009) MRI-based semiquantitative assessment of subchondral bone marrow lesions in osteoarthritis research. Osteoarthr Cartil 17(3):414-415 author reply 416-417

28. Kijowski R, Blankenbaker DG, Klaers JL, Shinki K, De Smet AA, Block WF (2009) Vastly undersampled isotropic projection steady-state free precession imaging of the knee: diagnostic performance compared with conventional MR. Radiology 251(1):185-194

\section{Publisher's Note}

Springer Nature remains neutral with regard to jurisdictional claims in published maps and institutional affiliations.

\section{Submit your manuscript to a SpringerOpen ${ }^{\circ}$ journal and benefit from:}

- Convenient online submission

- Rigorous peer review

- Open access: articles freely available online

- High visibility within the field

- Retaining the copyright to your article

Submit your next manuscript at $\boldsymbol{\nabla}$ springeropen.com 